\title{
INFECTIONS IN RECIPIENTS OF LIVER HOMOGRAFTS
}

\author{
Vincent A. Fulginiti, M.D., Robert Scribner, M.D., Carl G. Groth, M.D., Charles W. Putnam, \\ B.A., Lawrence Brettschneider, M.D., Spencer Gilbert, M.D., Ken A. Porter, M.D., and Thomas \\ E. Starzl, Ph.D., M.D.
}

\section{Abstract}

Seventeen patients received liver homografts between 1963 and May, 1968. The eight treated before July, 1967, died within 34 days; seven had progressive infections with gram-negative bacilli, Candida albicans and cytomegalovirus. The infections were similar to but more fulminating than those after renal homotransplantation.

In nine later cases, there was more discriminating donor selection, improved immunosuppression, and better organ preservation. In the first five of these nine patients, all infants, lobar hepatic gangrene apparently secondary to delayed right hepatic arterial thrombosis developed. Two died within a few days, two and three and a half months after transplantation. The three who did not die immediately subsequently had multiple bacteremias, fungemias and cytomegalovirus pulmonary infections. One of these children is alive twelve months after transplantation; the others died after four and a half and six months. In contrast, the last four patients, in whom septic liver infarctions were avoided, have been free of serious infections for two to five and a half months.

Infection is the most serious undesirable side effect of the immunosuppressive therapy that is necessary in clinical organ transplantation. This was well documented by Rifkind et al., ${ }^{1}$ who showed that 26 of the first 30 recipients of renal homografts at the University of Colorado Medical Center had infectious complications. In a later analysis, Hill et al. ${ }^{2}$ reported that infection had caused or contributed to more than two thirds of all the deaths that had occurred up to 1966 in our transplantation patients.

The present report presents information on the infectious complications seen in a series of liver transplantations. A number of bacterial, viral, fungal and protozoan diseases similar to those encountered after renal homotransplantation were seen. In addition, a more specific complication of hepatic homograft infection will be described that complicated the course or caused the death of the first five consecutive patients who survived for two months or more.

\section{METHODS}

\section{Case Material}

There were 19 patients. Sixteen received orthotopic homografts after total excision of their own diseased livers. ${ }^{3-5}$ In the other three auxiliary livers were transplanted to heterotopic locations at some distance from the host liver. ${ }^{6}$ One recipient of each kind of homograft died during operation and will not be considered further. The remaining 17 patients are divided into those treated before and after July, 1967. The eight recipients in the early series usually received livers that were badly damaged by ischemia. Moreover, they were treated with excessively

\footnotetext{
Copyright, 1968, by the Massachusetts Medical Society Printed in the U. S. A.

*From the departments of Surgery, Pediatrics and Pathology, University of Colorado School of Medicine and the Denver Veterans Administration Hospital, Denver, Col., and the Department of Pathology, St. Mary's Hospital and Medical School, London, England (address reprint requests to Dr. Starzl at Veterans Administration Hospital, 1055 Clermont St., Denver, Col. 80220).
} 
heavy immunosuppression. These errors were at least partially avoided in the later series of nine patients, who also profited from efforts at prospective histocompatibility matching.

Group 1 - early series (May, 1963, to May, 1967)—Six of the eight patients in this group had orthotopic homotransplantation, and the other two received auxiliary livers. Their ages ranged from 13 months to 67 years (average of 41 years). Seven were males, and a female. The diagnosis was hepatoma in four cases, cholangiocarcinoma in one, cirrhosis in two, and extrahepatic biliary atresia in the other.

Vascular reconstruction of the orthotopic homografts was anatomically normal. However, there were some variations in surgical technics. The first four recipients had staged procedures, hepatic mobilization being carried out one to 14 days before the actual transplantation; all these patients had choledochocholedochostomies and T-tube drainage. ${ }^{3}$ The other two patients had a one-stage operation, and provision for biliary drainage with cholecystoduodenostomy after ligation of the homograft and host common ducts. ${ }^{4,5}$

The two recipients of auxiliary homografts had emergency portacaval shunts for control of bleeding from esophageal varices, three and one days before auxiliary hepatic homotransplantation. The homografts were placed in the right paravertebral gutter. The hepatic arterial supply was taken from the aorta or hypogastric artery; portal inflow was from the external or common iliac veins. Biliary drainage was with Roux-Y cholecystojejunostomy. ${ }^{6}$

The organs employed were chilled as soon as possible after donor death, either by infusion through the portal vein with cold electrolyte solution or by total-body perfusion and cooling with a heart-lung machine. ${ }^{5}$ One of the livers was then temporarily stored and perfused in a hyperbaric oxygen chamber at $4^{\circ} \mathrm{C}$. In seven of the eight cases, there was evidence of massive or serious ischemic injury to the homograft as judged by serial liver-function measures after its transplantation.

All patients were treated with azathioprine and prednisone during the postoperative survival periods of six and a half to 34 days. Actinomycin $\mathrm{C}$ and local homograft irradiation were used in some cases. Heterologous antilymphocyte globulin (ALG) was also given to the last two recipients of orthotopic homografts in a regimen ${ }^{4,5}$ previously reported to be effective for renal homotransplantations.

Group 2 - later series (July, 1967, to May, 1968)-There were six females and three males. Seven of the nine patients were 14 to 23 months of age; the other two were 16 and 44 years. The indication for operation was extrahepatic biliary atresia in six cases and hepatoma in the other three. All received orthotopic liver transplantation and biliary reconstruction with cholecystoduodenostomy. In the first five cases (hereafter called Group 2A), the homografts were not fixed in position. Four of these patients died after 60, 105, 133 and 186 days; the remaining child is alive after 12 months. The next four recipients (Group 2B) had their homografts firmly fixed by resuture of the homograft and host triangular and falciform ligaments. These patients are all alive with follow-ups of two, three, four and five and a half months.

The patients in Group 2 differed from those in Group 1 in other important respects than their younger age. The donors were more carefully selected than in the previous attempts, had shorter terminal illnesses, had more vigorous attempts at maintenance of an effective circulation until almost the moment of death and had very prompt institution of post-mortem liver cooling. The margin of safety was further increased by interim preservation of the excised organs with the technic described by Brettschneider et al. ${ }^{7}$ All recipient operations were performed at one stage. 
Although the times from donor death to homograft revascularization in the recipients were from four to more than seven hours, all the organs provided satisfactory function. 4,5

Moreover, safer immunosuppression was given. Each patient received heterologous ALG for three weeks to four months after operation. Azathioprine was administered in less than half the doses as in Group 1. Prednisone therapy was somewhat reduced. In no case was a depressive effect noted on gamma globulin. Serial immunoglobulin determinations in one patient (OT-11) did not demonstrate any redistribution of $\operatorname{IgG}$, IgA or IgM.

\section{APPROACH TO INFECTIOUS PROBLEMS}

\section{Bacterial and Viral Studies}

On admission of the recipient, cultures were taken from the skin, trachea, urine and feces. During operation, additional specimens were cultured from the donor and recipient duodenum and the donor gallbladder bile. After transplantation, cultures were taken at least twice a week from the wounds, nose, throat, urine and feces, to provide a continuous record of the endogenous bacterial flora. In addition, blood cultures were obtained three or more times a week since it was soon learned that peripheral bacteremia could be the first sign of septic liver infarction.

In addition, weekly or biweekly swabs from the nasopharynx, throat, rectum and urine were obtained for virus isolation in the patients of Group 2. The swabs were immersed in veal infusion broth containing 0.5 per cent bovine serum albumin; $0.1 \mathrm{ml}$ was inoculated into monolayer cultures of human diploid fibroblasts (WI-38), primary rhesus-monkey-kidney cells and H-Ep ${ }^{2}$ cells. The cultures were observed daily for cytopathic effect and tested weekly for hemadsorption with guinea-pig erythrocytes. One blind passage was performed for each specimen in each system.

\section{Therapy}

Antibiotic therapy was similar in the cases of Group 1 and Group 2 but was more systematically designed in the latter series, to which the following remarks specifically apply. In two of the recipients in Group 2 there was enough time to attempt preoperative sterilization of the recipient gastrointestinal tract with a course of six and 48 hours respectively of oral neomycin, polymyxin, cloxacillin and mycostatin.

In all the patients of Group 2 methicillin or one of the other narrow-spectrum antistaphylococcal drugs was started intravenously before or during operation and continued afterward. In addition, agents were given that are usually effective against gram-negative organisms; these included ampicillin, cephalothin, kanamycin, chloramphenicol and polymyxin. The choice of antibiotics was made on the basis of prior cultures from the recipients' skin, trachea, urine and feces. In the event of growth from the intraoperative duodenal or bile cultures, an adjustment in drugs was made within 24 hours, on the basis of sensitivity data. Later on, antibiotics were frequently changed as dictated by the changing flora in the multiple culture sites.

\section{RESULTS}

\section{Hepatic Infections}

Group 1-Bacterial infection was not recognized during the six and a half to 34 days of posttransplantation life. In one (OT-7) a single microabscess was detected on histologic study. At autopsy the right lobe of the homograft in another (OT-5) contained a discrete 2-cm abscess (Table 1). Cholangitis was not present in any case. 
One patient had cytomegalovirus (CMV) in the homograft as well as in the lungs and gastrointestinal tract (Fig. 1).

Group 2A-The orthotopic homografts in these five consecutively treated infants (OT-8-12) were the site of massive septic liver infarction beginning two, 24, 25, 52 and 104 days posttransplantation. In each case the patients suddenly became febrile, with temperatures of 102.2 to $105.8^{\circ} \mathrm{F}\left(39\right.$ to $\left.41^{\circ} \mathrm{C}\right)$, exhibited increases in serum transaminases suggestive of massive liver necrosis, developed bacteremia with gram-negative bacilli and had large areas of absent isotope uptake in the right lobe of the homograft demonstrated by liver scans. ${ }^{4,5}$

Two of the children died within a few days despite efforts at debridement of what proved to be infarcted liver tissue. Two others were temporarily improved by such surgical intervention, only to die four and six months later of progressive liver failure; the immediate cause of death in one of the cases was intraperitoneal rupture of an unrecognized and undrained satellite liver abscess. Only one child (OT-8) recovered relatively completely. After debridement, the lost portion of liver regenerated, and now, 12 months later, she has only a small residual drainage tract connecting to the skin.

In all but one case the appearance of peripheral bacteremia coincided almost exactly with evidence of liver necrosis. In the exceptional patient (OT-12), bacteremia with aerobacterklebsiella was diagnosed 79 to 91 days after operation, without accompanying changes in liver chemical findings or alterations in the liver scans. Significant pneumonitis was present, with both cytomegalovirus and aerobacter-klebsiella isolated from tracheal aspirates. The bacteria disappeared from the bloodstream for 13 days with antimicrobial therapy, but then returned at the same time as a rapidly fatal infarction developed in the right lobe of the liver.

The agent infecting the liver in each of the five cases was a gram-negative bacterium: aerobacter-klebsiella species; Escherichia coli; and Bacteroides fragilis (Table 1). Two organisms were present in the homograft of one of the patients. In each the same organism was isolated from the blood. All three of the children who survived debridement had isolated subsequent positive blood cultures, often occurring at times when wound care had not been meticulous enough and pockets of fluid or pus had accumulated in the drainage tracts. At and after the onset of the complication, antibiotic therapy was selected and changed from time to time according to the growth in blood cultures and in the exposed liver tissue.

At the autopsies of the four unsuccessfully treated patients, there were similar findings. In each there was selective thrombosis of the right hepatic artery as reported elsewhere, ${ }^{5}$ and it is probable that the same thing occurred in the patient who is still alive; the consequence was total or subtotal lobar gangrene.

Cholangitis in the unaffected part of the liver was prominent in only one specimen (OT-12) from the child whose bacteremia had preceded overt hepatic infarction. In this case the intrahepatic duct system was found at autopsy to be dilated and full of a chalk-like material. Although the junction of the cystic and common ducts was patent, there may have been a partial biliary obstruction. The homograft of one other patient (OT-9) had extremely minimal histologic evidence of cholangitis.

Group 2B-Because of evidence cited elsewhere ${ }^{5}$ that the lobar dearterialization might be partially due to twisting or distortion of the right hepatic artery, the livers in the next four cases (OT 13-16) were firmly pinned to the diaphragmatic ligaments of the host. Septic hepatic infarctions have not occurred during the follow-up intervals of two, three, four and five and a half months. 
One of these patients (OT-15) had a severe rejection beginning on the sixth postoperative day. His bilirubin rose from normal to $14.4 \mathrm{mg}$ per $100 \mathrm{ml}$. There was fever, with minor concomitant rises in the serum transaminases. A liver scan showed a defect of the superior margin of the liver that returned to normal in a few days. One of the blood cultures at the peak of the rejection crisis contained Esch. coli.

In contrast to the patients in Group 2A, it was possible to discontinue all antibiotic therapy in four to six weeks without subsequent septic complications.

\section{Extrahepatic Sepsis}

The localization, etiology, timing, therapy and outcome of the infectious complications in all 17 patients are summarized in Table 1 .

Group 1-Seven of the eight patients in Group 1 had a total of 29 separate infections in the postoperative period of which only two, an Esch. coli urinary-tract infection (OT-5) and bronchiectasis (OT-7), existed before operation. The infectious complications were detected from the first to the last day of survival (maximum of 34) and at autopsy. Pneumonitis (seven episodes), peritonitis (four), esophagitis (three), urinary-tract sepsis (five), and bacteremia (three) were the most common diagnoses (Table 1).

Multiple foci of infection with a common organism were common. Thus, three patients had pneumonitis and bacteremia with the same micro-organism; two of the four cases of peritonitis were in patients who had the same culture in the trachea or bloodstream; and two of the five urinary-tract infections apparently led to bacteremia (Table 1).

It is noteworthy that pyogenic cocci were not responsible for any of the foregoing infections. Gram-negative enteric bacilli were the causative agents in 14 cases, including six of the seven pneumonias; the gram-negative bacteria were Esch. coli, aerobacter-klebsiella species, Pseudomonas aeruginosa and proteus species. There were also infections with C. albicans resulting in erosive esophagitis (OT-3, 5 and 6), panenteritis (AT-2), pyelonephritis (AT-2), pneumonitis (OT-2 and 3) and fungemia (OT-6). Cytomegalovirus (CMV) infestation of the lungs, gastrointestinal tract and liver were also seen (Table 1).

The completely uncontrollable septic complications in these patients were at least partially due to overdoses of immunosuppressive agents, particularly azathioprine. At some time during the postoperative course, five of the eight patients became leukopenic with total white-cell counts of less than 4000; in four of these cases the count fell to less than 2000.

Group 2A-Although leukopenia was avoided in these five patients, there were 25 episodes of extra-hepatic infection (Table 1). However, these could be effectively treated and were not responsible for the unfavorable outcome. The primary cause of the four deaths was the septic hepatic infarctions described earlier.

The five children had a total of 16 bouts of bacteremia due to gram-negative organisms that did not coincide with, but occurred just before or at some time after the septic hepatic infarctions. When the sinus tracts into the liver were cultured in the patients who survived debridement, they sometimes contained the same bacteria as those contemporaneously found in the blood, but they often did not. Because of this frequent lack of association, the sporadic bacteremias due to gram-negative organisms have been classed as nonhepatic in origin.

Nevertheless, it is highly likely that the homograft was the responsible locus since recurrent bacteremias have not characterized the convalescence of patients in Group 2B. At least two other infections were thought to have been hematogenously "seeded" by these bacterial 
showers, whatever their origin. One was a hip abscess due to $B$. fragilis (OT-9) and the other was an Esch. coli meningitis (OT-11).

There were only two examples of bacterial pneumonia, in contrast to the experience in Group 1 , these being gram-negative infections. CMV was isolated from the lungs of three children (OT-9, 10, and 12) at the time of autopsy; during life the same virus had been repeatedly isolated from tracheal aspirates in two of these patients, of whom one (OT-12) had clinically significant pneumonitis that, as noted above, had an important bacterial component; in addition, the same post-mortem specimen contained Pneumocystis carinii. P. carinii was also found in the autopsy lung of patient OT-9, although symptoms or x-ray signs of pneumonia had not been present before death. In three of the patients in this group fungemia developed, two with $C$. albicans and one with an unidentified yeast. All three cleared with antifungal therapy (amphotericin B or 5-fluorocytosine ${ }^{*}$ ), but in one (OT-9) silent residual abscesses of the cerebellum were found at autopsy.

Group 2B-None of these four patients has had a significant gram-negative bacterial infection with follow-up periods of two to five and a half months. One (OT-16) had two blood cultures of Staphylococcus albus (coagulase negative) one month after operation that cleared with penicillin therapy. In two of the patients (OT-14 and 15) herpes-simplex virus has been cultured from the throat at weekly intervals (Table 1).

\title{
DISCUSSION
}

Many of the infectious complications encountered in this series of 17 liver transplantations were similar to but of greater severity than those previously reported after transplantation of the kidney. ${ }^{1,2}$ Common features after both kinds of operation included the predisposition to pneumonitis, the tendency for localized infections to become quickly generalized and the frequent etiologic role of gram-negative bacteria, fungi and less common micro-organisms of normally low pathogenicity. The development of qualitatively comparable problems in recipients of renal and hepatic homografts was hardly surprising since essentially the same kind of immunosuppressive therapy was used for both groups.

The role of these drugs in lowering host reactivity to certain ambient micro-organisms is undoubted. However, the precise mechanism of this effect is not known. In patients treated with renal homotransplantation, Rifkind et al. ${ }^{1}$ noted that steroid-induced diabetes mellitus, hypogammaglobulinemia and granulocytopenia were frequently associated with fatal infections. The liver recipients with infectious complications almost never had the first two findings. The immunoglobulins were usually normal or increased. There was only one example of steroid-induced diabetes and that in a patient (OT-14) who has never had an infection. In contrast, the occurrence of granulocytopenia was an extremely common and grave finding in the first patients provided with liver homografts; florid leukopenia was observed in five of the first eight hepatic recipients.

\begin{abstract}
Almost certainly, the bone-marrow depression in these cases was induced by overdoses of azathioprine. The quantities of this drug administered to the first liver patients were guided by previous experience with renal recipients. The latter patients in the period between 1962 and 1966 averaged between 2.1 and $4.1 \mathrm{mg}$ per kilogram of body weight per day of azathioprine for the first two postoperative months. Such doses in liver recipients proved to be lethal as was proved in the Group 1 hepatic series. The patients in Group 2 were given less than $1 \mathrm{mg}$ per kilogram per day. These small doses in combination with prednisone and heterologous ALG provided good protection of the homografts from rejection., 4
\end{abstract}

\footnotetext{
*Roche Company, Professional Service Department, 212 Oxford, New York, N.Y.
} 
The avoidance of leukopenia did not prevent infections in the patients of Group 2, although these infections were not as overwhelming. The frequency, type and etiology of infections in the latter series were comparable to those seen in children who have a congenital lack of cellular immunity with or without impaired immunoglobulin synthesis. ${ }^{8,9}$ The functional impairment of the small and medium lymphocyte is probably the common link, owing in the first instance to congenital and in the other to pharmacologic factors. More precise future studies of the role of the lymphocyte in defense against gram-negative bacilli, fungi, viruses of the herpes group, and $P$. carinii will be awaited with interest.

The influence of antibiotic therapy in promoting the overgrowth of opportunistic microorganisms also was probably a crucial factor. The way in which antibiotics were used varied from series to series. For example, the patients of Group 1 who had poor initial homograft function were enfeebled in the postoperative period, and had to have protracted ventilatory support, bladder catheterization and total bed rest. These requirements probably contributed to the high proportion of lung and urinary-tract infections that required treatment; with persistent antibiotic therapy, infections with opportunistic organisms followed. This cumulative effect of one complication upon another is a well recognized sequence after the less complex procedure of renal homotransplantation. ${ }^{10}$

The patients of Group 2A were in better general condition after operation. However, they received systemic antibiotic therapy for months in an effort to prevent or control hepatic sepsis; in two of these patients (OT-11 and 12) intestinal antibiotics were also used. The consequence was the emergence of bacterial populations that were resistant to the antimicrobial agents used. In addition, infection with fungi occurred probably as a result of their becoming more firmly established in the respiratory and gastrointestinal tracts. This phenomenon has been observed repeatedly in nontransplant patients ${ }^{11-13}$ and is also reflected in the etiology of infectious deaths in recipients of renal homografts. ${ }^{2}$

Despite such adverse circumstances, aggressive attempts at diagnosis are of more than academic interest since some of these resulting infections, including those caused by fungi, cytomegalovirus and $P$. carinii, can be treated by intensive courses of experimental or uncommonly used drugs or with immune globulin. ${ }^{14-16}$ The value of endotracheal aspirations, blood cultures and esophagoseopy should be emphasized in view of the repeatedly significant findings in such samples.

It is evident from the foregoing remarks that the highly specific complication of partial hepatic homograft infarction seen in Group 2A made the total problem of infectious-disease control an exceedingly difficult and often an eventually hopeless undertaking because of the consequent necessity for long-term antibiotic therapy. The pathogenesis and prevention of hepatic infarction has been discussed elsewhere. ${ }^{5}$ Briefly, the gangrene of the right lobe may have had a partial mechanical origin caused by distortion of the right hepatic artery, to which reduced blood flow and homograft swelling at the time of rejection may have contributed. Thereafter, it was not surprising that the necrotic tissue became the focus of an acute and lifethreatening infection. The fixation of the liver homograft in a desirable position apparently eliminated this dread complication in subsequent cases.

With the advent of major hepatic parenchymal necrosis in five cases (OT 8-12), the homograft was invaded by bacteria that are normally found in the gastrointestinal tract, just as has been shown in patients not given immunosuppressive drugs in whom liver abscesses nevertheless develop; under the latter circumstances, ascending cholangitis is found in about 40 per cent of cases. ${ }^{17}$ Whether the secondary bacterial invasion of the homografts was through the biliary tract or the blood supply (particularly the portal vein) or both is not known in human patients and could not be determined in laboratory studies of pig and dog homotransplantation by 
Brettschneider et al. ${ }^{18}$ In the latter experiments, the infection of liver tissue occurred at about the same time as the identical bacteria appeared in all the portal structures as well as the duodenum.

The experience in the most recent cases suggests that the problem of regional ischemic necrosis can be avoided. It now remains to be seen to what extent more subtle kinds of chronic hepatic infection will jeopardize the long-term course. It is encouraging to note that significant cholangitis has been found in the autopsy homograft of only the one patient (OT-12) who may have had partial biliary-tract obstruction. Conceivably, low-grade cholangitis may prove to be tolerable on a chronic basis. An analogous situation has already been shown to be possible in kidney homografts. A number of patients are known to have survived for years after renal homotransplantation with persistent bacteriuria, with or without continuous antibiotic treatment. ${ }^{10,19}$

\section{Acknowledgments}

Supported by grants (AM-06344, HE-07735, AM-07772, AI-04152, FR-00051, FR-00069, FO5-TW-1154, AM-12148 and AI-AM-08898) from the United States Public Health Service.

\section{REFERENCES}

1. Rifkind D, Marchioro TL, Waddell WR, Starzl TE. Infectious diseases associated with renal homotransplantation. J.A.M.A 1964;189:397-407.

2. Hill RB Jr, Dahrling BE II, Starzl TE, Rifkind D. Death after transplantation: analysis of sixty cases. Am. J. Med 1967;42:327-334. [PubMed: 4381194]

3. Starzl TE, et al. Homotransplantation of liver in humans. Surg., Gynec. \& Obst 1963;117:659-676. [PubMed: 14100514]

4. Starzl TE, et al. Extended survival in 3 cases of orthotopic homotransplantation of human liver. Surgery 1968;63:549-563. [PubMed: 4171413]

5. Starzl TE, et al. Orthotopic homotransplantation of human liver. Ann. Surg. (in press)

6. Halgrimson CG, et al. Auxiliary liver transplantation: effect of host portacaval shunt: experimental and clinical observations. Arch. Surg 1966;93:107-118. [PubMed: 5327725]

7. Brettschneider L, et al. Use of combined preservation techniques for extended storage of orthotopic liver homografts. Surg., Gynec. \& Obst 1968;126:263-274.

8. Miller ME, Schieken RM. Thymic dysplasia: separable entity from "Swiss agammaglobulinemia.". Am. J. M. Sc 1967;253:741-750. [PubMed: 5338315]

9. Haworth JC, Hoogstraten J, Taylor H. Thymic alymphoplasia. Arch. Dis. Childhood 1967;42:40-54. [PubMed: 6019467]

10. Starzl, TE. Experience in Renal Transplantation (Contributing authors: Joseph H. Holmes and others). Philadelphia: Saunders; 1964.

11. Karl RC, Mertz JJ, Veith FJ, Dineen P. Prophylactic antimicrobial drugs in surgery. New Eng. J. Med 1966;275:305-308. [PubMed: 5328634]

12. Johnstone FRC. Assessment of prophylactic antibiotics in general surgery. Surg., Gynec. \& Obst 1963;116:1-10.

13. Petersdorf RG, et al. Study of antibiotic prophylaxis in unconscious patients. New Eng. J. Med 1957;257:1001-1009. [PubMed: 13483877]

14. Cangir A, Sullivan MP, Sutow WW, Taylor G. Cytomegalovirus syndrome in children with acute leukemia: treatment with floxuridine. J.A.M.A 1967;201:612-615.

15. Robbins JB, Miller RH, Arean VM, Pearson HA. Successful treatment of Pneumocystis carinii pneumonitis in patient with congenital hypogammaglobulinemia. New Eng. J. Med 1965;272:708713. [PubMed: 14261461]

16. Rifkind D, Faris TD, Hill RB Jr. Pneumocystis carinii pneumonia: studies on diagnosis and treatment. Ann. Int. Med 1966;65:943-956. [PubMed: 5332224] 
17. Sherman JD, Robbins SL. Changing trends in casuistics of hepatic abscess. Am. J. Med 1960;28:943950. [PubMed: 14445885]

18. Brettschneider L, et al. Specific bacteriologic problems with canine orthotopic liver transplantation. Arch. Surg 1968;97:313-322. [PubMed: 4872484]

19. Prout GR Jr, Hume DM, Lee HM, Williams GM. Some urological aspects of 93 consecutive renal homotransplants in modified recipients. J. Urol 1967;97:409-425. [PubMed: 4163805] 


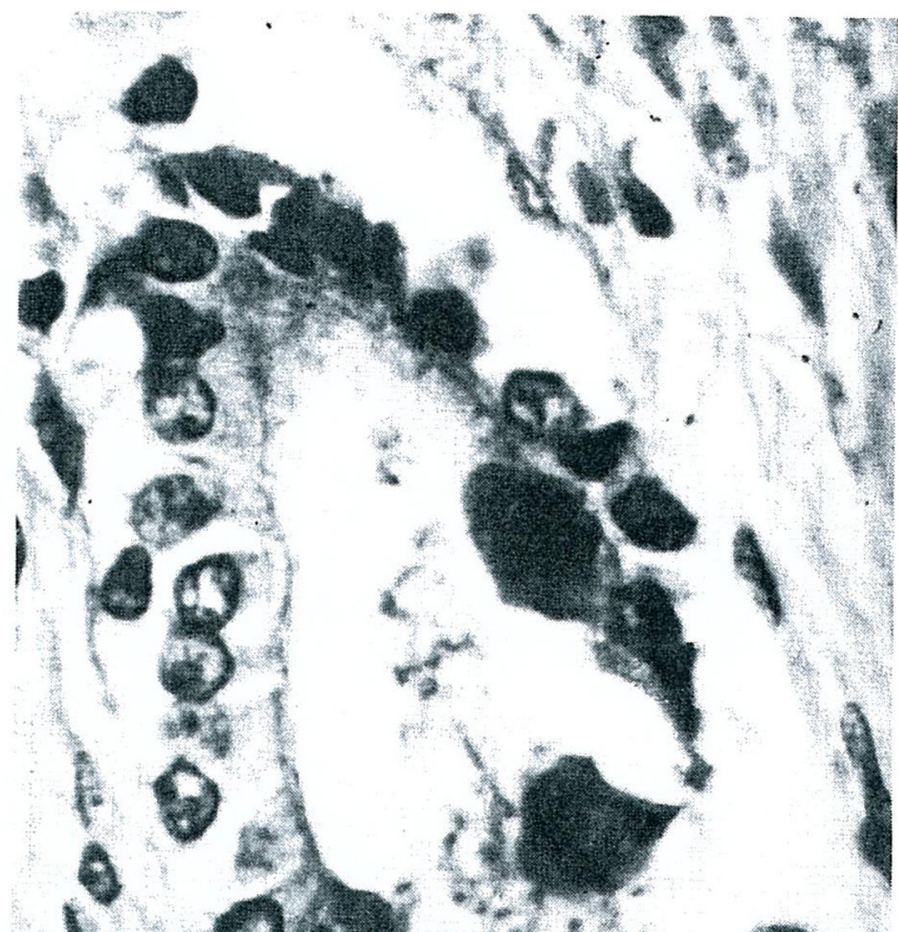

Cytomegalovirus in a Small Bile Duct of the Auxiliary Homograft of a Patient (AT-2), 34 Days after Operation (Hematoxylin and Eosin Stain X200). 


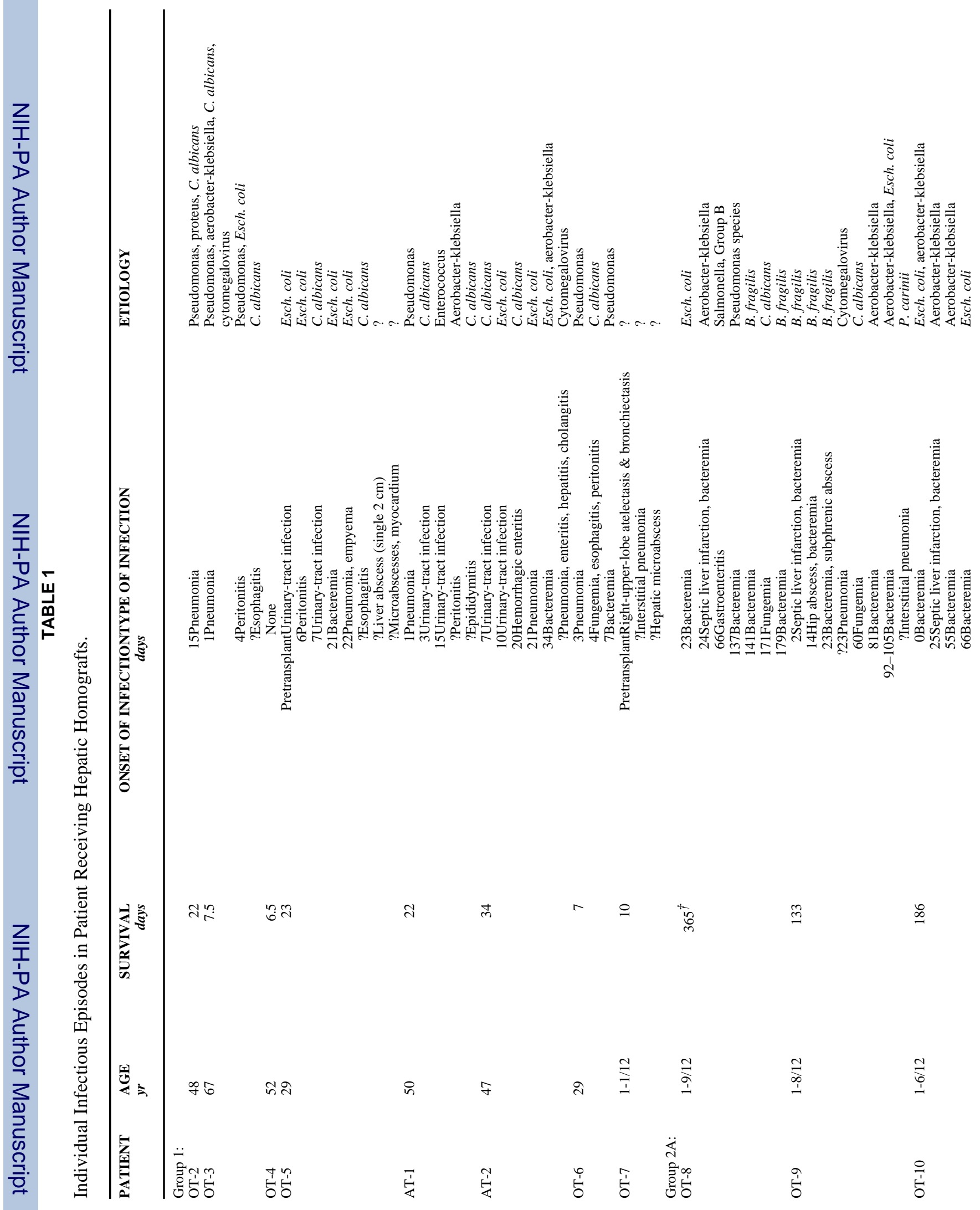




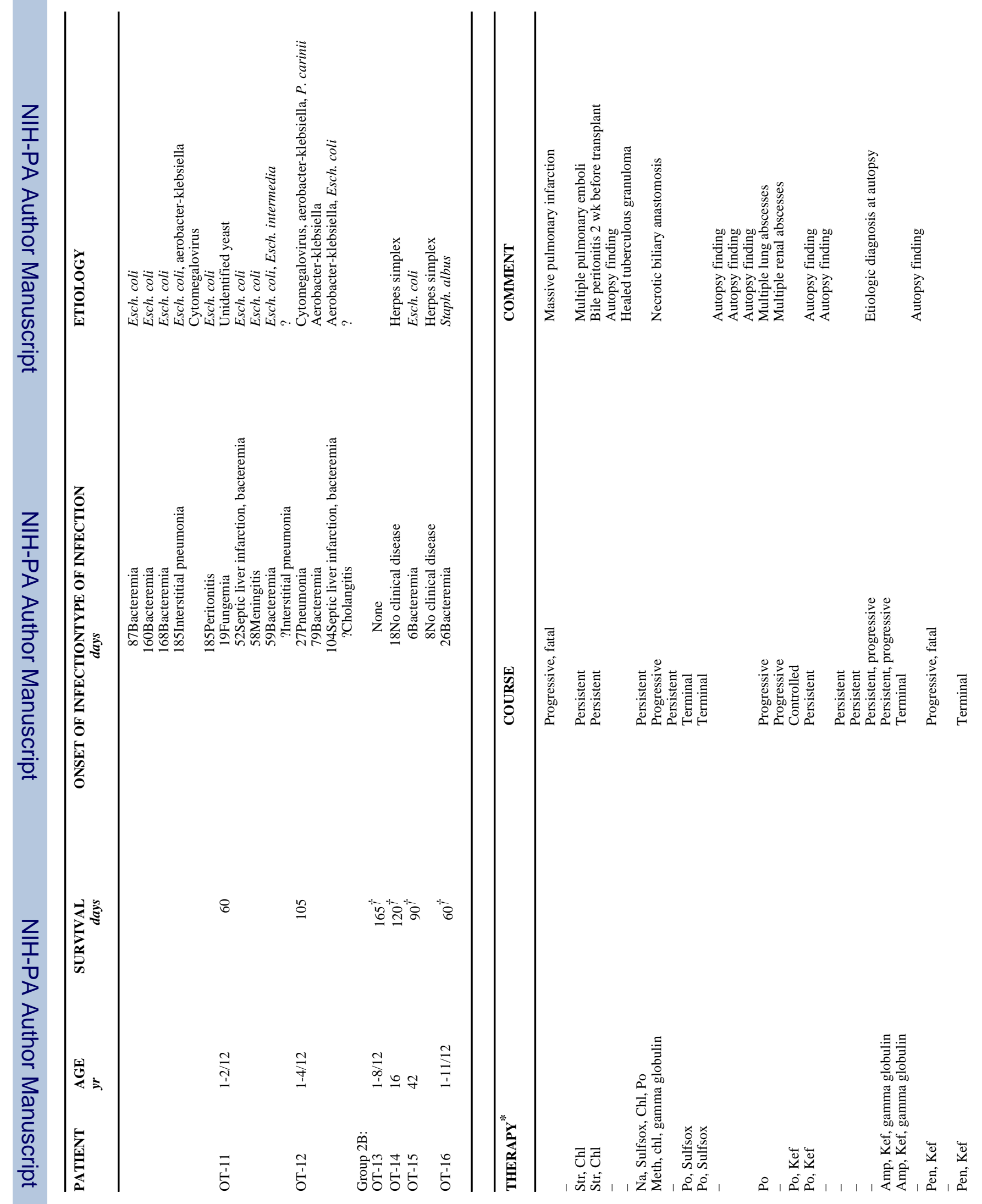


Fulginiti et al.

Page 13

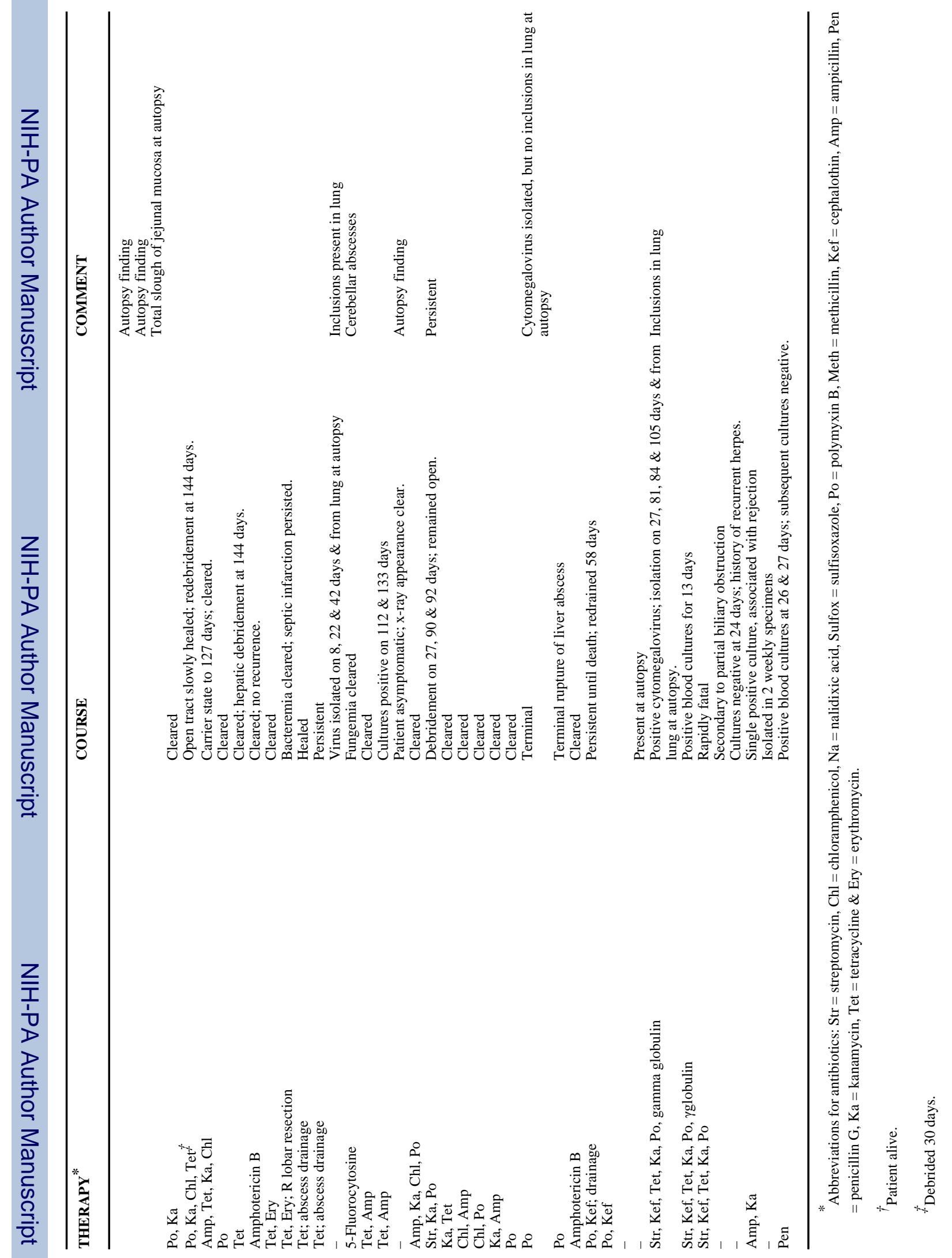

N Engl J Med. Author manuscript; available in PMC 2009 November 3. 